\title{
2 Browser
}

Lernziele

In diesem Kapitel lernen Sie, dass

Browser Werkzeuge für den Zugriff

auf die Dienste des WWW sind, die

man für seine Bedürfnisse gut an-

passen kann.

Inhalt dieses Kapitels:

- Vergleich der gängigen Browser

- Personalisierung der Browser

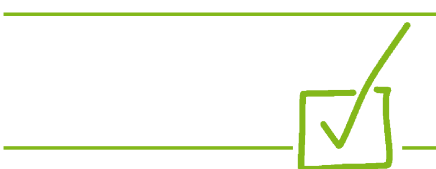

Tipp

Da die behandelten Browser

Windowsprogramme sind, gelten

für sie auch die „Shortcuts“

(=Tastenkombinationen), wie für

alle Windowsprogramme. Sehr

wichtig in diesem Zusammenhang

ist die Kombination Strg/Ctrl+f.

Damit öffnen Sie jeweils ein kleines

Suchfenster, mit dessen Hilfe Sie

die gerade angezeigte Webseite

mit Hilfe eines Stichwortes durch-

suchen können. Wie oft hat man

lange textlastige Seiten oder gar

verschachtelte, überladene Web-

angebote. Viel besser als sich die

Augen zu verderben: Suchen!

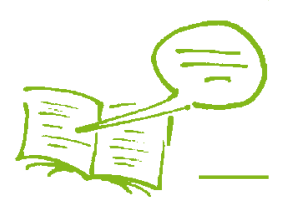

Ein Tutorial zum Einstieg in den Firefox [firefox-anleitung.net]
Der Browser ist das Werkzeug, mit dem Sie Internetseiten und -dienste aufrufen und verwenden, daher sollten Sie sich einen Überblick darüber verschaffen, welcher Browser für Ihre persönliche Arbeitsorganisation am besten geeignet ist.

Dies hängt zum einen vom Betriebssystem Ihres Computers ab, und zum anderen kann es natürlich auch Beschränkungen am Arbeitsplatz bei der freien Auswahl des Browsers geben. Damit Sie aber für sich einschätzen können, welchen oder welche Browser Sie in optimaler Weise für sich verwenden möchten, bieten wir an dieser Stelle einen kurzen Vergleich der meistgenutzten Browser. Da Browser, wie so vieles in diesem Bereich, ständigen Veränderungen unterworfen sind, hat ein Vergleich nur einen begrenzt lange Gültigkeit, kann aber in jedem Fall als Orientierung dienen.

\subsection{Vergleich}

\subsubsection{Firefox}

Der Firefox zählt zu den in Deutschland am häufigsten genutzten Browsern und zeichnet sich durch seine vergleichsweise starke Personalisierbarkeit aus. Im Gegensatz zu den Browsern IE (Internet Explorer) und Chrome handelt es sich bei dem Browser Firefox um eine Open Source Software, an deren Weiterentwicklung viele Programmierer weltweit arbeiten. Betreut wird dieses Projekt von der Mozilla Foundation, die verschiedene Webprojekte betreut. Der Firefox ist sowohl für Windows als auch für Linux einzusetzen.

Firefox ist über Erweiterungen, sogenannte Add-ons und Plug-ins, personalisierbar. Auch die Browsereinstellungen erlauben eine starke Anpassung des Browsers an die persönlichen Vorlieben.

\subsubsection{Internet Explorer}

Der IE ist der hauseigene Browser der Firma Microsoft und auf jedem Rechner mit Windows-Betriebsystem vorinstalliert. Dadurch bedingt hat er eine hohe Verbreitung, hat bei den Nutzern aber in den letzten Jahren stark an Beliebtheit eingebüßt. Er ist in der Regel etwas langsamer als die anderen Browser, weniger personalisierbar und weist immer wieder Probleme bei der Anwendung verschiedener Web 2.0-Dienste auf. Es kann passieren, dass manche Dienste oder Funktionen im IE nicht verwendbar sind. Auch der IE kann mit Erweiterungen personalisiert werden, hier steht allerdings im Vergleich zum Firefox eine weitaus geringere Anzahl an Erweiterungen zur Verfügung.

\subsubsection{Chrome}

Der Chrome ist der Browser aus dem Hause Google und arbeitet dementsprechend besonders gut mit den verschiedenen Google-Diensten zusammen. Hier zeigt der Chrome eine deutlich höhere Integration dieser Dienste als die anderen Browser. Der Chrome ist durch Webapps und Erweiterungen personalisierbar. Zur Zeit seiner Einführung auf dem Markt (als Beta) standen vor allem die Datenschutzrichtlinen von Google-Chrome in der Kritik. Dies hat sich aber seit Einführung der Vollversion ge- 
legt, und Chrome ist inzwischen ebenfalls sehr verbreitet im Einsatz. Er kann leicht samt Add-ons für den Betrieb in einer Konfiguration auf mehreren Geräten synchronisiert werden.

\subsubsection{Andere Browser}

Im Bereich des Betriebssystems Windows sollte man noch die beiden Browser Opera und Safari anführen. Safari ist ähnlich anpassbar wie Firefox und Chrome, Opera nicht, bietet aber ein ganz eigenes Profil der Handhabung. Opera ist sehr schnell und bietet auch Browser für Smartphones an, so dass man sich hier nicht umgewöhnen muss. Safari ist hauptsächlich der Browser für Apple-Computer, seit einigen Jahren aber auch für Windows verfügbar.

Diese Graphik zeigt anhand der Daten von StatsCounter die Verbreitung der Browser im weltweiten Vergleich Januar 2012 bis März 2012:

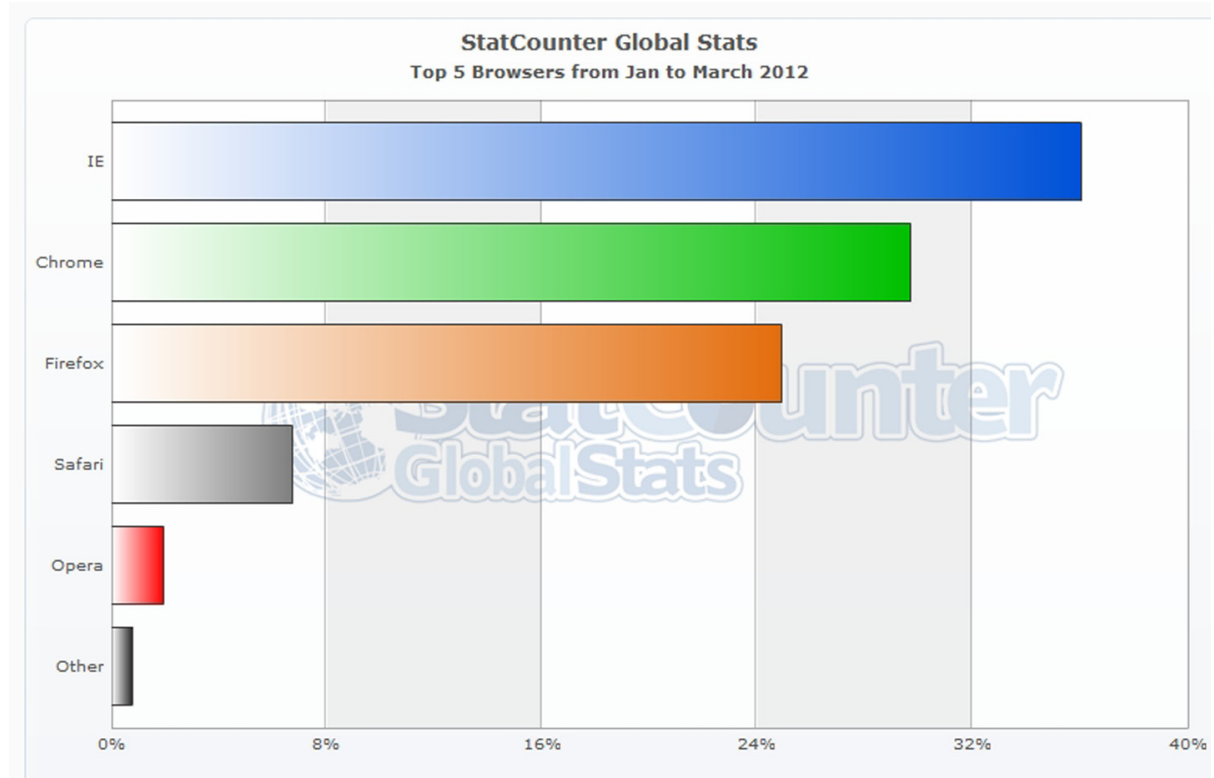

Quelle: http://gs.statcounter.com/\#browser-ww-monthly-201201-201203-bar

\subsection{Personalisieren}

Jeder der genannten drei Browser lässt sich mehr oder eben weniger stark an Ihre persönlichen Bedürfnisse anpassen, sprich: personalisieren. Wichtig für die reibungslose Nutzung der verschiedenen Internetdienste ist, dass Sie Ihren Browser jeweils aktuell halten. Bei Windows-Betriebssystemen ist zu beachten, dass der IE - auch wenn Sie Ihn gegebenenfalls nicht oder nicht oft nutzen - aktuell zu halten ist, da sonst Sicherheitslücken bei der Nutzung des Internets entstehen können. Windows lässt bestimmte Prozesse bei der Verbindung mit dem Internet im Hintergrund über den IE verwalten.

Erwähnt werden sollte auch, dass Personalisierung eine Schattenseite hat: Wenn Sie auf Ihrem Gerät den Browser stark an Ihre Bedürfnisse angepasst haben und dann bei bestimmten Gelegenheiten - beispielsweise bei Veranstaltungen und Schulun- 
gen - also dann, wenn es eigentlich darauf ankommt, souverän mit dem Browser zu arbeiten - mit ungewohnten Ansichten eines Browsers arbeiten müssen, kann das einen schlechten Eindruck hinterlassen.

\subsubsection{Firefox}

\section{Einstellungen}

Mit den Einstellungen konfigurieren Sie die Grundfunktionen des Browsers. Warum diese unter Extras in der obersten Befehlsleiste des Browsers zu finden sind, wird uns für immer unerklärlich bleiben ... Jedenfalls, wenn Sie „Extras“ angeklickt haben, öffnet sich ein kleines Extrafenster mit dem voreingestellten Registerblatt „Allgemein“ und etlichen weiteren Registerblättern. Hier können Sie die Grundfunktionen Ihres Browsers einstellen.

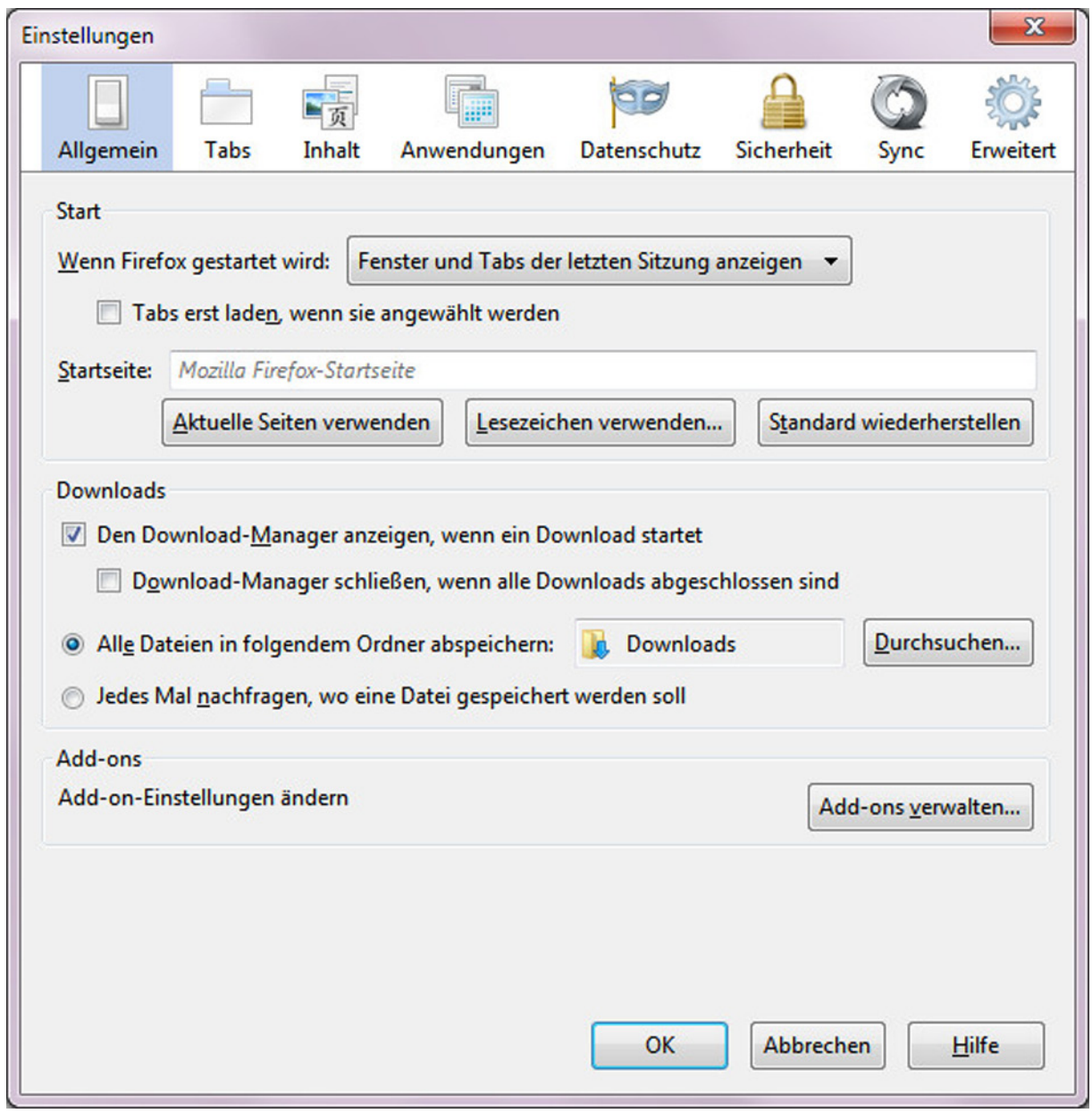


Empfehlenswerte Einstellungen

\section{Registerblatt „Allgemein“}

- „Startseite anzeigen“

- In das Feld „Startseite“ passen mehrere Webseiten. Wenn Sie mit immer denselben paar Seiten arbeiten, dann lohnt es sich, diese beim Start des Browsers gleich zu laden.

- Downloads: Am besten immer nachfragen lassen, wo man eine herunterzuladende Datei ablegen möchte, das sorgt für eine adäquate Ablage.

\section{Registerblatt „Tabs“}

- „Statt neuem Fenster neuen Tab öffnen“ sorgt dafür, dass Sie den Überblick auf dem Desktop behalten.

\section{Registerblatt „Inhalt“}

- „Pop up“-Fenster blockieren - wenn dies auch nicht immer gelingt. Lieber im Bedarfsfall eine Ausnahmeregel festlegen.

\section{Registerblatt „Anwendungen“}

- Hier können Sie festlegen, welcher Dateityp auf einer Webseite mit welchen Anwendungen geöffnet werden soll.

\section{Registerblatt „Datenschutz“}

- Sie müssen selbst abschätzen, ob Sie eine Chronik - eine chronologische Liste der von Ihnen besuchten Webseiten - benötigen und wie sicher Ihr Computer ist. Wenn die Chronik angelegt wird, kann jede/r die von Ihnen besuchten Seiten nachsehen.

- Bei den Cookies sollten zumindest jene von Drittanbietern, das sind meist die Werbeeinblendungen auf einer Webseite, nicht akzeptiert werden.

\section{Registerblatt „Sicherheit“}

- Alle vorgeschlagenen Warnungen sind sinnvoll.

- Passwörter sollte man zumindest mit einem Masterpasswort verschlüsseln, sonst geht die Benutzerfreundlichkeit zu Lasten der Sicherheit.

\section{Registerblatt „Sync“}

- Siehe den Tippkasten

\section{Registerblatt „Erweitert“}

- Hier sollten Sie durch Setzen/Entfernen der Häkchen selbst herausfinden, wie diese Optionen sich auswirken und welche Konfiguration Ihnen passt.

- Unter „Netzwerk“ ist der Cache zu finden, dessen Größe man einstellen kann. Hier ist auch der wichtige Knopf zum Leeren des Caches: Wenn Sie den Eindruck haben, dass dargestellte Webseiten sich aktualisiert haben könnten, ohne dass das dargestellt wird, sollten Sie den Cache leeren!
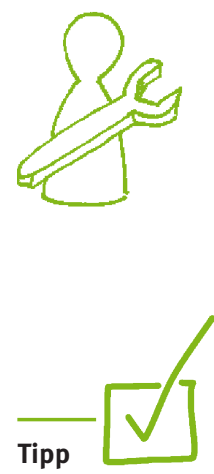

Wer den Firefox auf mehreren Geräten verwendet und nicht überall mit dieselben Einstellungen arbeiten möchte, hat mehrere Optionen:

1. Mit Hilfe von Firefox-Sync die Lesezeichen und Einstellungen synchronisieren - aber nicht die Add-ons.

2. Mit dem Add-on Xmarks [www.xmarks.com] Bookmarks und - optional - Passwörter zu synchronisieren, auch zwischen verschiedenen Browsern.

3. Firefox auf dem USB-Stick installieren: Mobile Versionen gibt es unter [portableapps.com/de]. 


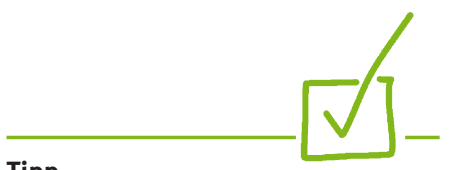

Tipp

Ganz am oberen Rand ist hinter „Hilfe“ oft freier Platz, insbesondere bei breiten Bildschirmen. Wenn Sie diesen nutzen wollen, können Sie ihn mit der Lesezeichenleiste belegen. Das geht so: „Anpassen“-Menü laden, wie im letzten Abschnitt besprochen, dann die Lesezeichenleiste links anklicken, festhalten, auf die Fläche hinter "Hilfe“ ziehen und fallen lassen. Jetzt werden die Lesezeichenlinks oben angezeigt fertig!

\section{Anpassen der Kopfleiste}

Sie können sich die obere Zone Ihres Browsers an Ihre Bedürfnisse anpassen. Klicken Sie irgendwo in die Fläche mit der rechten Maustaste hinein und wählen Sie in dem aufklappenden Menü ganzunten „Anpassen“aus. Dann poppt ein Extrafenster auf

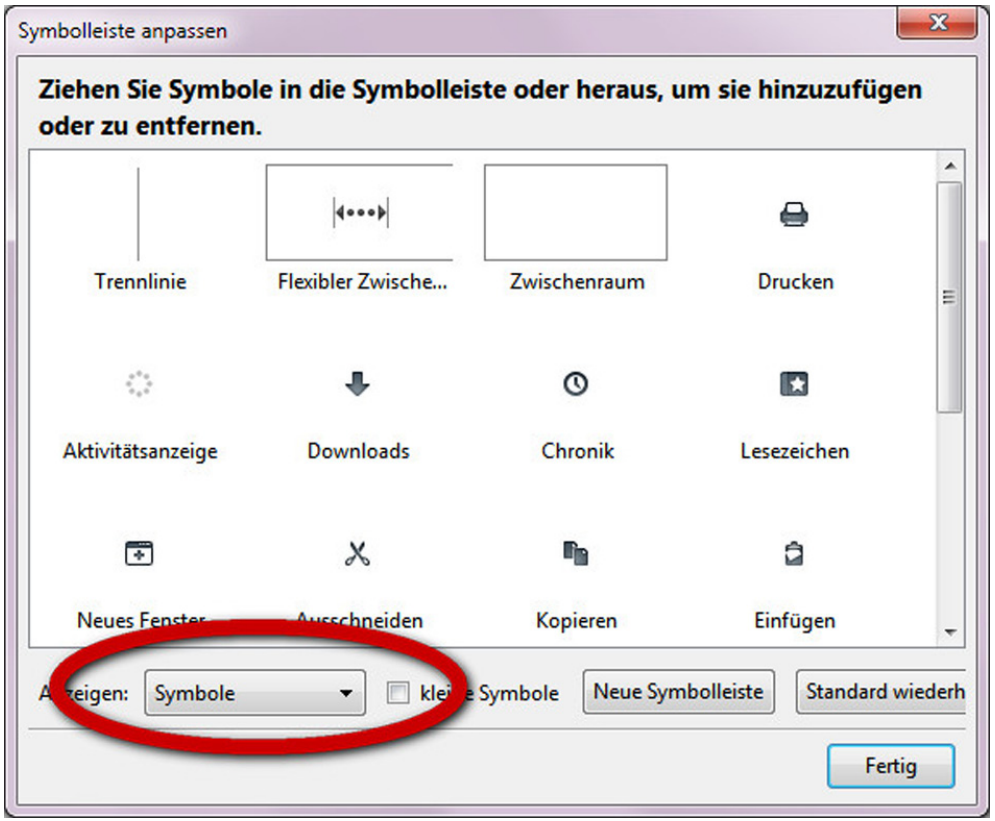

und die ganze Kopfzone wird bearbeitbar. Lassen wir erst einmal das Extrafenster beiseite, versuchen Sie, das Suchfenster, das standardmäßig rechts angesiedelt ist, auf derselben Ebene nach links zu ziehen. Anklicken mit der linken Maustaste, festhalten, hinüberziehen und am gewünschten Ort fallen lassen. Sehr gut! Ebenso können Sie jetzt auch die Icons, die bereits auf der Leiste sind, hin- und herschieben. Wenn Sie mehr benötigen, sehen Sie sich im Extrafenster um und ziehen Benötigtes nach oben an den gewünschten Ort. Nicht benötigte Icons ziehen Sie ins Extrafenster und lassen sie dort fallen. Keine Angst, Sie können nichts kaputt machen, denn im Extrafenster gibt es die Option „Standard wieder herstellen“!

Bei den Icons sehen Sie auch solche, die nicht nur zum Firefox, sondern auch zu Add-ons gehören. Auch diese können Sie anordnen, wie es Ihnen passt.

Wenn Sie einzelne Zonen temporär ausschalten möchten, dann klicken Sie wieder mit rechts in die Kopfzone und können dann Häkchen wegnehmen bei den Leisten, die Sie gerade nicht benötigen und Häkchen setzen, wenn Sie etwas benötigen.

\section{Lesezeichenleiste}

Die wichtigsten Links für Ihre Arbeit sollten Sie immer nahebei haben, am besten nur einen Klick entfernt. Dazu haben Sie die Option einer Lesezeichenleiste oberhalb des Browserfensters, auf welche Sie alle Links platzieren können, die Sie immer wieder verwenden.

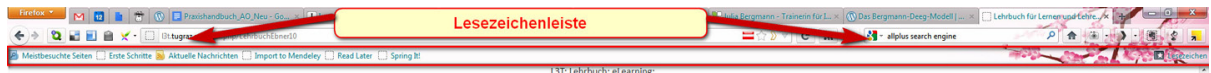

Die Lesezeichenleiste können Sie verstecken und wieder laden lassen, indem Sie einen Rechtsklick in den Kopfbereich des Browsers machen, worauf dann ein Pop-up- 
Fenster erscheint, in dem Sie dann ein Häkchen bei „Lesezeichenleiste“ setzen oder wegnehmen können, je nachdem, ob sie sichtbar sein soll oder nicht. Wenn Sie, wie im Tippkasten beschrieben, die Lesezeichenleiste an den oberen Rand verschoben haben, können Sie so die leere Leiste wegnehmen.

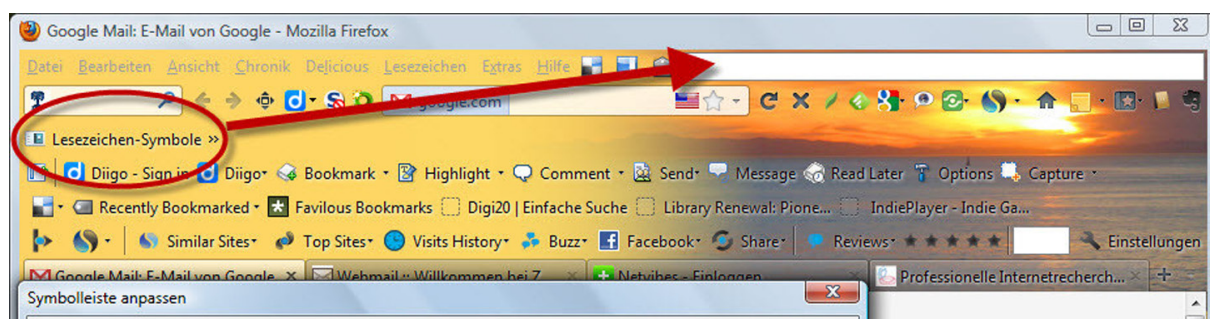

Sie können die Ordner und Lesezeichen auf der Lesezeichenleiste leicht per „drag \& $d r o p$ " verschieben, indem Sie das Lesezeichen anklicken, festhalten, ziehen und am gewünschten Ort loslassen.

\section{Add-ons}

Den Firefox-Browser können Sie nun mit so genannten Add-ons, also Zusätzen, ergänzen. Add-ons bieten Ihnen eine Erweiterung der Funktionalität Ihres Browsers nach Ihren Bedürfnissen. Die Anzahl der verfügbaren Add-ons ist sehr groß und es ist zu empfehlen, nicht zu viele auszusuchen, weil sie den Browser, je mehr installiert sind, beim Start und im Betrieb empfindlich verlangsamen. Es gilt also eine Balance zwischen Gewinn an Funktionalität und der verringerten Geschwindigkeit des Browsers zu finden. Probieren Sie also ruhig Add-ons aus, zögern Sie aber auch nicht, sie wieder zu deaktivieren, falls Sie sie nicht oft benötigen!

Ein Add-on ist wie eine Art Rucksack. Sie können ihn aufziehen, aber auch wieder abnehmen und ohne ihn spazieren gehen. So ist es auch hier. Add-ons sind Erweiterungen, die bei Deinstallation keine Spuren im Browser hinterlassen, da sie nur ein Zusatz waren.

Wie kommen Sie nun zu den Add-ons? Sie klicken oben auf der Befehlsleiste „Extras“ und dann „Add-ons“ an und sind dann beim „Add-ons“-Manager, welcher Ihnen die bereits installierten („Erweiterungen“) und die optionalen Add-ons („Addons suchen“) auflistet.

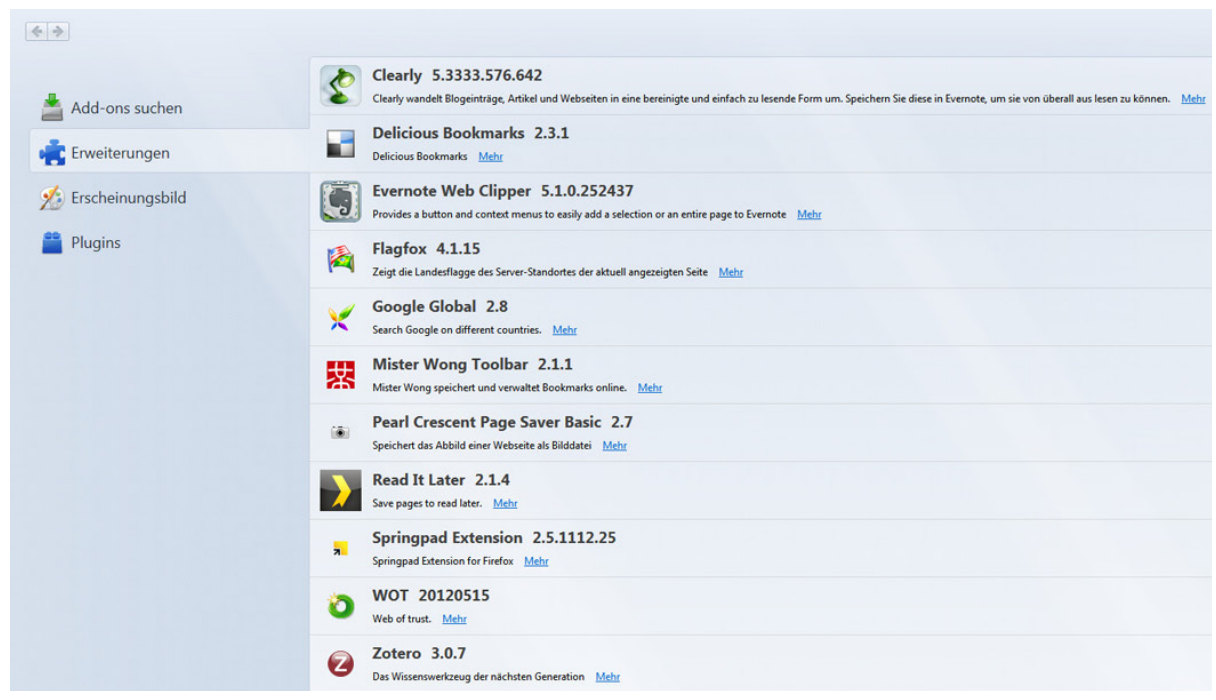

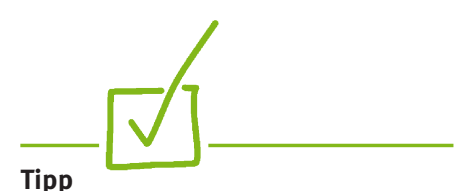

Wenn auf der Lesezeichenleiste mehr Bookmarks sind als Platz vorhanden ist, dann können Sie

- die Bezeichnungen der einzelnen Bookmarks abkürzen. Klicken Sie mit der rechten Maustaste auf den jeweiligen Eintrag, wählen Sie beim Aufklappmenü „Eigenschaften“ aus und kürzen Sie den Namen.

- Auf dieser Leiste kann man auch Ordner anlegen: Rechtsklick auf die Lesezeichenleiste, „neuer Ordner" anklicken, Namen angeben und fertig! Beim Klicken auf ein Ordnersymbol öffnet sich dann ein Aufklappmenü mit allen Bookmarks, die diesem Ordner zugeordnet sind. Diese Zuordnung erreichen Sie ganz einfach durch Anklicken einer Bookmark auf der Leiste, festhalten und auf den Ordner ziehen. Ebenso ordnen Sie die Reihenfolge innerhalb eines Ordners oder auf der Leiste. 


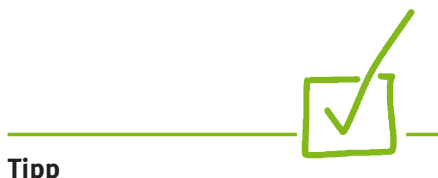

FEBE (Firefox Environment Backup Extension) bietet Ihnen die Möglichkeit, Ihre Sammlung von Add-ons als Back-up zu sichern, um Sie in einem Schwung (bei z. B. einer kompletten Neuinstallation Ihres Rechners) neu installieren zu können. FEBE kann nicht nur lhre Add-ons als Back-up sichern, sondern auch Ihre Bookmarks, Themes und persönliche Einstellungen.
Letztere sind auch gleich über diesen Link zu sehen: [addons.mozilla.org/de/firefox/extensions]. Bei den „vorgestellten Erweiterungen“ sind schon einige interessante Add-ons dabei, wichtiger ist es aber, links die für Ihre Arbeit relevanten Kategorien $\mathrm{zu}$ durchforsten, die Ihnen die Add-ons themenorientiert präsentieren. Besonders interessant sind natürlich die Kategorien „Datenschutz und Sicherheit“, „Lesezeichen“ und „RSS-Feeds ...“. Sie können bei jeder Kategorie die links oben angezeigten Optionen Empfohlen/Beliebteste/Am höchsten bewertet anklicken und bekommen jeweils eine Auswahl angezeigt. Oft gibt es auch für die in diesem Buch vorgestellten Programme Add-ons, die die Bedienung komfortabler machen.

Da es eine Unzahl an Erweiterungen gibt, haben wir Ihnen in einer Checkliste die von uns täglich verwendeten Add-ons zusammengestellt.

Add-ons für Firefox

„Musts“

- $\quad$ Adblock Plus - blockiert die Werbung, die in Webseiten via Banner (= graphische Werbeanzeigen) angezeigt wird

- Xmarks - Bookmarks und Passwörter (falls gewünscht) zwischen verschiedenen Geräten synchronisieren

- $\quad$ RSS Icon - in der Befehlszeile des Browsers wird angezeigt, ob ein RSS-Feed von der gerade geladenen Webseite angeboten wird. Abonnieren durch Klicken auf das Icon.

- Google Shortcuts - Googles Dienste schnell laden

- Shareaholic - die aktuell dargestellte Webseite schnell in soziale Dienste posten

- $\quad$ Add to Search Bar - so können Sie Suchoptionen von Suchmaschinen, Katalogen etc. diesem kleinen Suchfenster oben im Browser schnell hinzufügen

Gute Werkzeuge

- Delicious Extension - zum schnellen Einpflegen in die soziale Bookmarksammlung Delicious

- $\quad$ Diigolet - Bookmarks in Diigo einpflegen, highlighten, Notizen schreiben, Webseiten sichern

- Zotero - eine ganze Literaturverwaltung als Browser-Extension

- Evernote Web Clipper - Notizen in Evernote hinterlegen

- $\quad$ Quick Fox Notes - Notizen im Browser hinterlegen, synchronisierbar mit Hilfe von Diigo

- $\quad$ NoScript - blockiert unerwünschte Skripte auf Webseiten, kann konfiguriert werden

- Flashblock - gibt Inhalte mit Flash erst frei, wenn Sie es erlauben

- Clearly - erlaubt das Ausblenden von störenden Webseiten-Elementen wie Werbung, Zusatzinformationen etc.

- Ghostery - zeigt Ihnen an, an wie viele und welche Datensammeldienste Ihre Nutzung der aktuellen Webseite gemeldet wird

- Stealthy - anonym über einen Proxy-Server surfen

- World of Trust - Webseitenbewertung

- feedly - RSS Reader

- Similar Web - ähnliche Webseiten anzeigen lassen

- Stumble Upon - ähnliche Webseiten und Community mit Webseiten-Bewertung

- Screengrab - Bildausschnitte vom Browser auswählen und als Bild abspeichern

- Sage - ein RSS-Reader in der Sidebar

- EPUB-Reader - zeigt eBooks diesen Formats im Browser korrekt an

- Flagfox - zeigt die Landesflagge des Serverstandortes der aktuell angezeigten Webseite an

Wenn Sie ein Add-on installieren wollen, klicken Sie auf den blauen „Jetzt herunterladen"-Knopf, dann installiert es sich von selbst. Sie müssen nach Aufforderung den Browser neu starten und dann müsste ein neues Icon neben der Adresszeile zu sehen 
sein, welches das Add-on repräsentiert. Manche Add-ons freilich setzen sich auch höflich ganz unten auf die Leiste ...

Ein Ärgernis ist in letzter Zeit, dass in kurzer Zeitfolge neue Versionen des Browsers herauskommen und dann die Add-ons noch nicht angepasst und in Folge dessen deaktiviert sind. Unserer Erfahrung nach dauert es aber nur kurze Zeit, bis die Anpassungen durch die Programmierer der einzelnen Erweiterungen erfolgt sind.

\section{Themes}

Die so genannten Themes haben keine Funktionalität, sie geben Ihrem Browser lediglich ein anderes Aussehen. Jedoch ist unsere Erfahrung, dass ein freundliches Theme das Arbeiten doch erheblich aufhellt.

\section{Plug-ins}

Plug-ins sucht man meist nicht, sondern sie werden angefordert, wenn auf einer Webseite eine Anwendung ist und der Browser das nicht selbst öffnen kann. So findet man in schon länger benutzten Browsern viele Plug-ins wie PDF-Viewer, Flash-Player etc. Es gibt auch eine ganze Anzahl Plug-ins, die Sie in Ihre Schnellsuche integrieren können, wie z.B. die Schnellsuche in Amazon, Wikipedia, dem Wörterbuch Leo uvm. Sie können die Schnellsuche anpassen, indem Sie auf den kleinen Pfeil vor dem Logo klicken und im Menü „Suchmaschinen verwalten“ auswählen und anklicken.

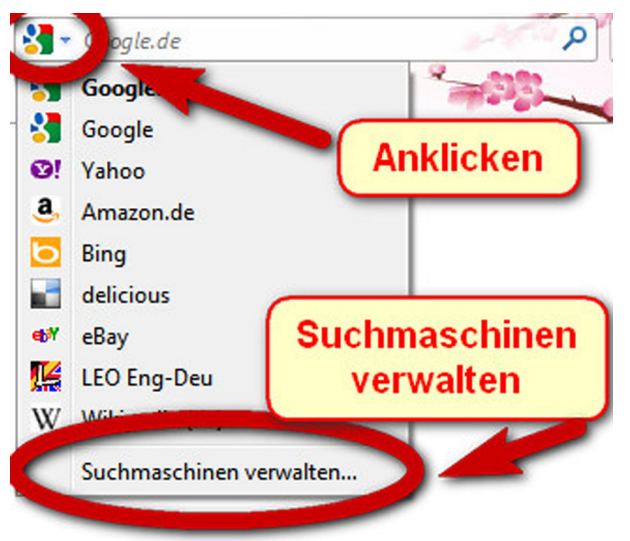

Hier können Sie neue Suchmaschinen hinzufügen - wenn die gewünschten nicht aufgeführt sind, können Sie mit dem Add-on „Add to Searchbar“ arbeiten oder die Reihenfolge der angezeigten Suchmaschinen verändern.

\subsubsection{Internet Explorer IE}

\section{Einstellungen}

Wir hatten oben bereits erwähnt, dass man den Internet Explorer aktuell halten sollte, weil über ihn die Art und Weise, wie sich der Computer mit dem Internet verbindet, mit konfiguriert wird. Diese Konfiguration finden Sie in der Kopfzone des Browsers ganz rechts, wenn Sie auf das Zahnrad-Icon klicken. Jetzt erscheint das Aufklappme-

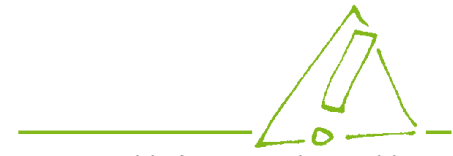

Von verschiedenen Suchmaschinen und Diensten werden so genannte „Toolbars“ angeboten - beispielsweise von Google, Yahoo!, Diigo. Das kann Vorteile haben, wenn man einen Dienst wirklich intensiv nutzt. Im Allgemeinen aber sollte man sich das überlegen, denn die Toolbar nimmt in der Regel mehr Platz weg, als einem lieb ist. - Sie haben aber auch die Option, verschiedene Toolbars zu installieren und dann jeweils durch „Anpassen“ ein- und auszuschalten. 
nü für die Einstellungen des Browsers, und sie klicken auf „Internetoptionen“. Ein Extrafenster poppt auf mit verschiedenen Registerkarten, deren wichtigste Funktionen die folgende Checkbox anzeigt.

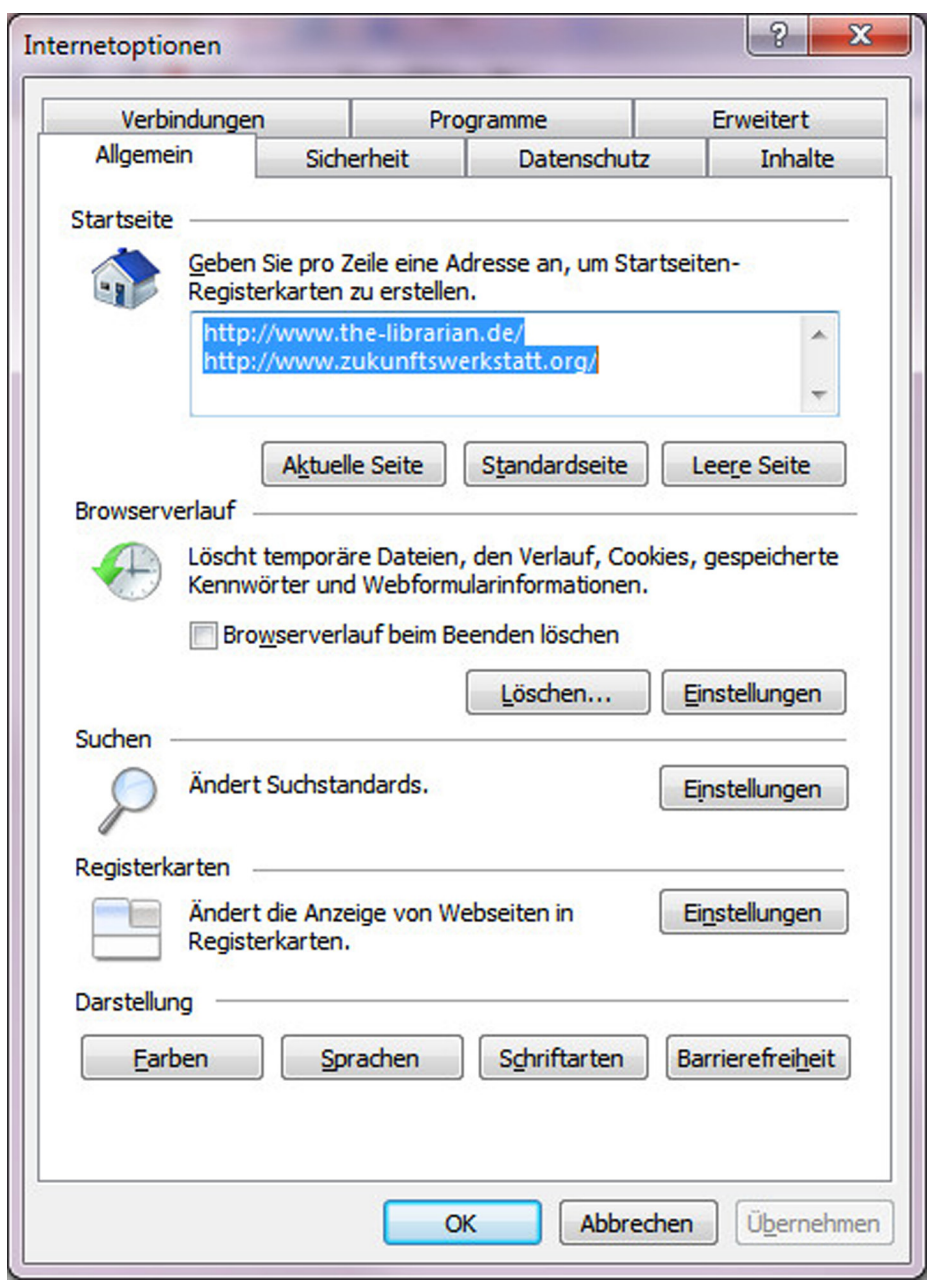


Registerkarte „Allgemein“

- $\quad$ Auch hier haben Sie die Option, unter „Startseite“ mehrere Webseiten anzugeben, die Sie ständig nutzen und die gleich beim Start des Browsers geladen werden sollen.

- Bei Browserverlauf haben Sie die Option, die Chronik oder den „Verlauf“ bei Beenden des Browsers löschen zu lassen. Unter „Einstellungen“ können Sie festlegen, wie groß der Cache ist und ob er bei Neustart des Browsers aktualisiert werden soll. Vorsicht: Hier lauern gleich zwei Dinge, die nerven können. Ein großer Cache führt zu oft inaktuell angezeigten Seiten. Und wenn man die Aktualisierungsoption aktiviert, hat man zwar den ersten Effekt bekämpft, wundert sich dann aber, warum im Hintergrund so viele Prozesse ablaufen, die den Browser verlangsamen. Leeren Sie ab und zu den Cache, aber nur dann, wenn Sie nicht die volle Leistung des Rechners benötigen.

\section{Registerkarte „Datenschutz“}

- Die Internetzone zumindest auf „Mittel“ stellen.

- Die Abfrage des physischen Ortes ausschalten.

- Den Popupblocker einschalten oder mit Hilfe von „Einstellungen“ konfigurieren.

\section{Registerkarte „Inhalte“}

- $\quad$ Filter sind nur dann in Erwägung zu ziehen, wenn auch Kinder auf Ihrem Computer zugange sind.

\section{Registerkarte „Sicherheit“"}

Einstellung für die verschiedenen „Zonen“. Hier können Sie jeweils individuell einstellen.

- Internet - bei uns „mittelhoch“

- lokales Intranet - „niedrig“

- Vertrauenswürdige Sites - „Mittel“ bis „niedrig“

- Eingeschränkte Sites - „hoch“

\section{Registerkarte „Verbindungen“}

- Hier wird die Grundkonfiguration für die Verbindung mit dem Internet eingegeben.

\section{Registerkarte „Programme“}

- Den Punkt „Add-ons“ beschreiben wir unten gesondert.

- Wenn Sie hier unter „Internetprogramme“ auf „Programme festlegen“ klicken, wird auch hier die für den ganzen Computer geltende Konfigurationsdatei „Standardprogramme“ aufgerufen, die festlegt, welche Dateitypen mit welchem Programm geöffnet/bearbeitet werden.

\section{Registerkarte „Erweitert“}

- Hier können Sie durch Setzen/Wegnehmen von Häkchen den Browser konfigurieren.

- Was nicht selbst erklärend ist, sollten Sie entweder ausprobieren oder über die Hilfe die benötigte Information abfragen.

- Sie können nichts kaputt machen, sondern die Standardeinstellungen durch „Zurücksetzen“ wieder herstellen. 


\section{Anpassen der Kopfleiste}

Sie können den IE anpassen, indem Sie in die Kopfzone rechts hineinklicken und verschiedene Leisten hinzu- oder abwählen.

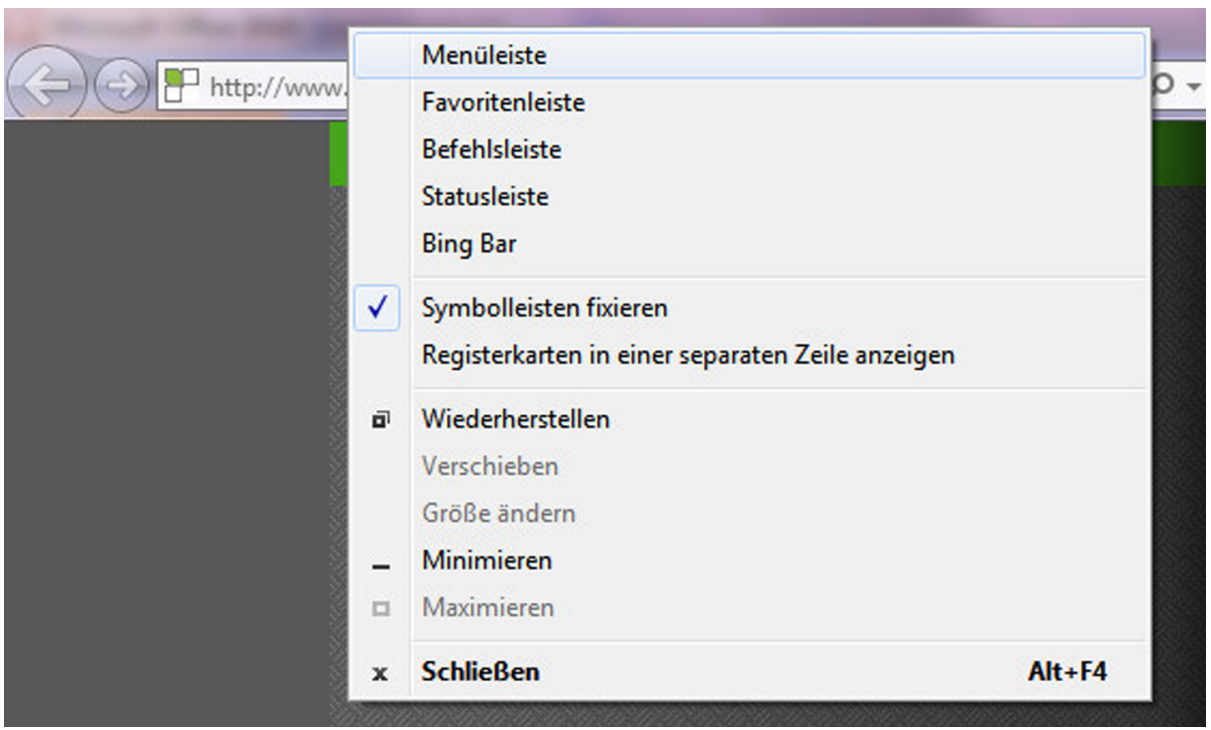

Beispielsweise die Befehlsleiste, mit der Sie vieles, was wir oben anhand der „Internetoptionen“ gezeigt haben, schnell einstellen und verändern können. Hier finden Sie dann auch die Icons der aktivierten Add-ons.

\section{Add-ons}

Durch Klick auf das Zahnrad rechts und dann „Add-ons verwalten“ kommen Sie zu einem Extra-Fenster, in welchem die geladenen, aktiven und deaktivierten Add-ons angezeigt werden.

\begin{tabular}{|c|c|c|c|c|c|}
\hline \multicolumn{2}{|l|}{ Add-Ons verwalten } & & & & $\cos =0$ \\
\hline \multicolumn{6}{|c|}{ Internet Explorer-Add-Ons anzeigen und verwalten } \\
\hline Add-On-Typen & Name & Herausgeber $\hat{~}$ & Status & Ladezeit & Navigation... \\
\hline - Symbolleisten und Erweiterungen & \multicolumn{5}{|l|}{ Adobe Systems, Incorporated } \\
\hline Suchanbieter & Adobe PDF Link Helper & Adobe Systems, Incorpo... & Aktiviert & $0,05 \mathrm{~s}$ & $0,00 \mathrm{~s}$ \\
\hline 目Schnellinfos & Hewlett-Packard Company & & & & \\
\hline \multirow{9}{*}{ Q Tracking-Schutz } & HP Print Enhancer & Hewlett-Packard Comp... & Deaktiviert & & \\
\hline & HP Smart BHO Class & Hewlett-Packard Comp... & Deaktiviert & & \\
\hline & FE HP Smart Web Printing ein- od... & Hewlett-Packard Comp... & Aktiviert & & \\
\hline & HP Smart Web Printing & Hewlett-Packard Comp... & Deaktiviert & & \\
\hline & Kaspersky Lab & & & & \\
\hline & IEVkbdBHO Class & Kaspersky Lab & Deaktiviert & & \\
\hline & FilterBHO Class & Kaspersky Lab & Deaktiviert & & \\
\hline & [ Virtuelle Tastatur & Kaspersky Lab & Aktiviert & & \\
\hline & $\mathbf{K}$ Links untersuchen & Kaspersky Lab & Aktiviert & & \\
\hline Momentan geladene Add-Ons & \multicolumn{5}{|c|}{ Lenovo Information Products (Shenzhen) Co, Ltd - } \\
\hline \multicolumn{6}{|c|}{ Wählen Sie ein Add-On aus, um den Status zu ändern oder Details anzuzeigen. } \\
\hline \multicolumn{6}{|c|}{$\begin{array}{l}\text { Weitere Symbolleisten und Erweiterungen suchen... } \\
\text { Weitere Informationen zu Symbolleisten und Erweiterungen }\end{array}$} \\
\hline
\end{tabular}


Neue Add-ons können Sie auf der IE-Gallery [www.iegallery.com/de-DE/addons oder durch Klick auf das Lupen-Symbol im Befehls-/Suchfenster und dann auf „Hinzufügen"] ansehen und bei Gefallen laden.

Plug-ins werden bei Bedarf installiert.

\section{Lesezeichenleiste}

Die Lesezeichen heißen beim IE „Favoriten“ und das Hinzufügen von Lesezeichen sowie die Verwaltung derselben können Sie durch Klicken auf das Sternchen neben dem Zahnradsymbol herholen. Wenn Sie diese in der Nähe haben wollen, klicken Sie auf das Sternchen und dann links auf den kleinen grünen Pfeil, dann öffnen sich die Lesezeichen in einer Seitenleiste. Falls das zu viel Platz erfordert, können Sie auch hier eine Lesezeichenleiste einrichten:

- klicken Sie mit der rechten Maustaste in die Kopfzone des Browsers

- hier können Sie wieder die unterschiedlichsten Leisten, Toolbars und Add-ons aktivieren und deaktivieren

- aktivieren Sie die „Favoritenleiste“

- hier können Sie Lesezeichen per „drag \& drop“ aus einer Webseite (Link anklikken - festhalten - auf die Leiste ziehen - loslassen) importieren und per „drag \& drop" ordnen

- Ordner auf der Lesezeichenleiste können Sie über die Favoritenverwaltung (gelben Stern anklicken - Favoritenverwaltung) anlegen

\subsubsection{Chrome}

\section{Einstellungen}

Aussehen und Funktionen können Sie beim Chrome-Browser durch Klicken auf das Schraubenschlüssel-Symbol rechts in der Kopfzone des Browsers verändern. Die wichtigen beiden Punkte bei dem Aufklappmenü, das sich dann öffnet, sind „Tools“ und „Einstellungen“.

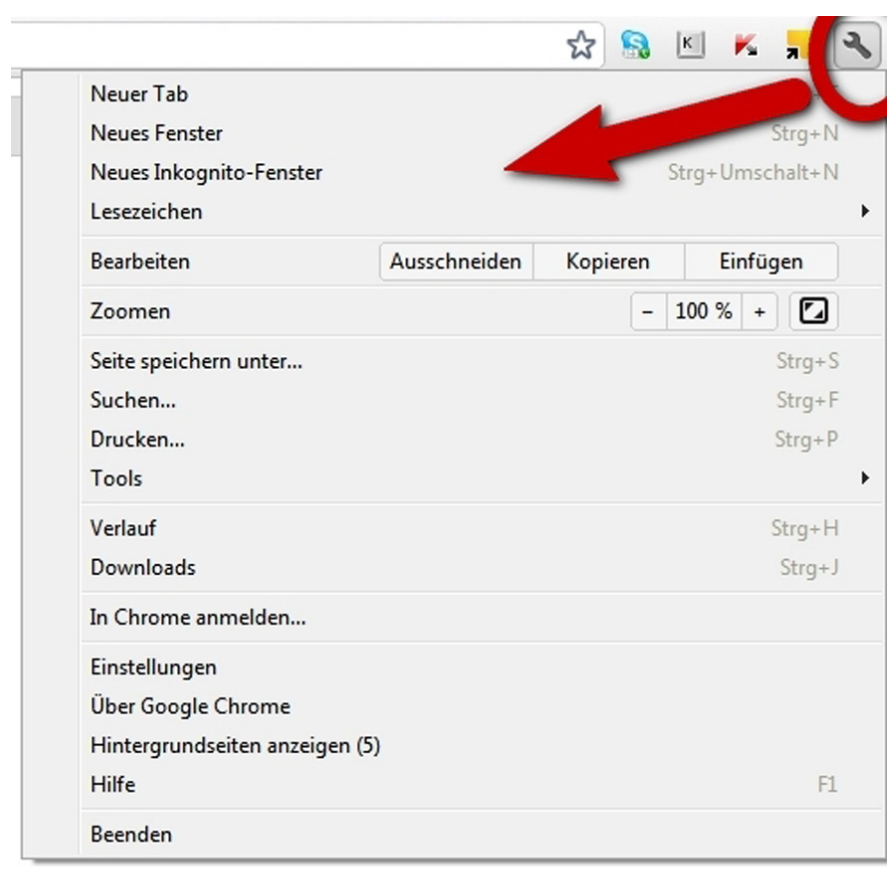

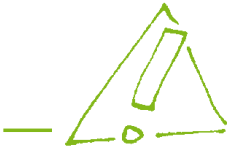

Add-ons deaktivieren, wenn Sie sie länger nicht benötigen! 
Sie werden feststellen, dass sich manches, was unter den einzelnen Hauptpunkten aufgelistet ist, überlappt.

Die Punkte, die darunter aufgezählt sind, erklären sich meist von selbst. Bei den Tools ist der Punkt „Browserdaten“ wichtig, wo Sie hinterlegte Daten wie z. B. Verlauf und Cookies löschen können. Bei den Einstellungen finden Sie unter „Privates“ die Möglichkeiten, den Browser über verschiedene Geräte hinweg zu synchronisieren (ein Google-Konto ist dafür die Voraussetzung), die Downloadoptionen zu beeinflussen und anderes.

Die Icons auf der Kopfleiste können Sie ganz einfach mit Hilfe von drag \& drop versetzen und gruppieren.

\section{Anpassen der Kopfleiste}

Im Kopfbereich des Chrome gibt es nur eine Symbolleiste: die Lesezeichenleiste. Sie wird nicht, wie bei den anderen Browsern, durch Rechtsklick in den Kopfbereich geladen, sondern durch Klick auf das Schraubenschlüsselsymbol > Lesezeichen > Lesezeichenleiste. Auch hier können Sie einfach durch drag \& drop Lesezeichen hinzufügen, Ordner anlegen und so die Leiste zu einem Produktivitätstool machen, auf dem Sie alle täglich benutzten Links in Reichweite zur Verfügung haben.

\section{Add-ons}

Die wichtigsten Add-ons, die für den Firefox-Browser verfügbar sind, sind auch auf dem Chrome-Browser im Angebot und über das Werkzeug-Symbol rechts > Tools > Erweiterungen anzusehen. Sie können die hier angezeigten installierten Erweiterungen leicht deaktivieren und wieder aktivieren. Ganz unten ist ein Link „Weitere Erweiterungen herunterladen“ zu finden, wo Sie dann Erweiterungen recherchieren oder browsen können.

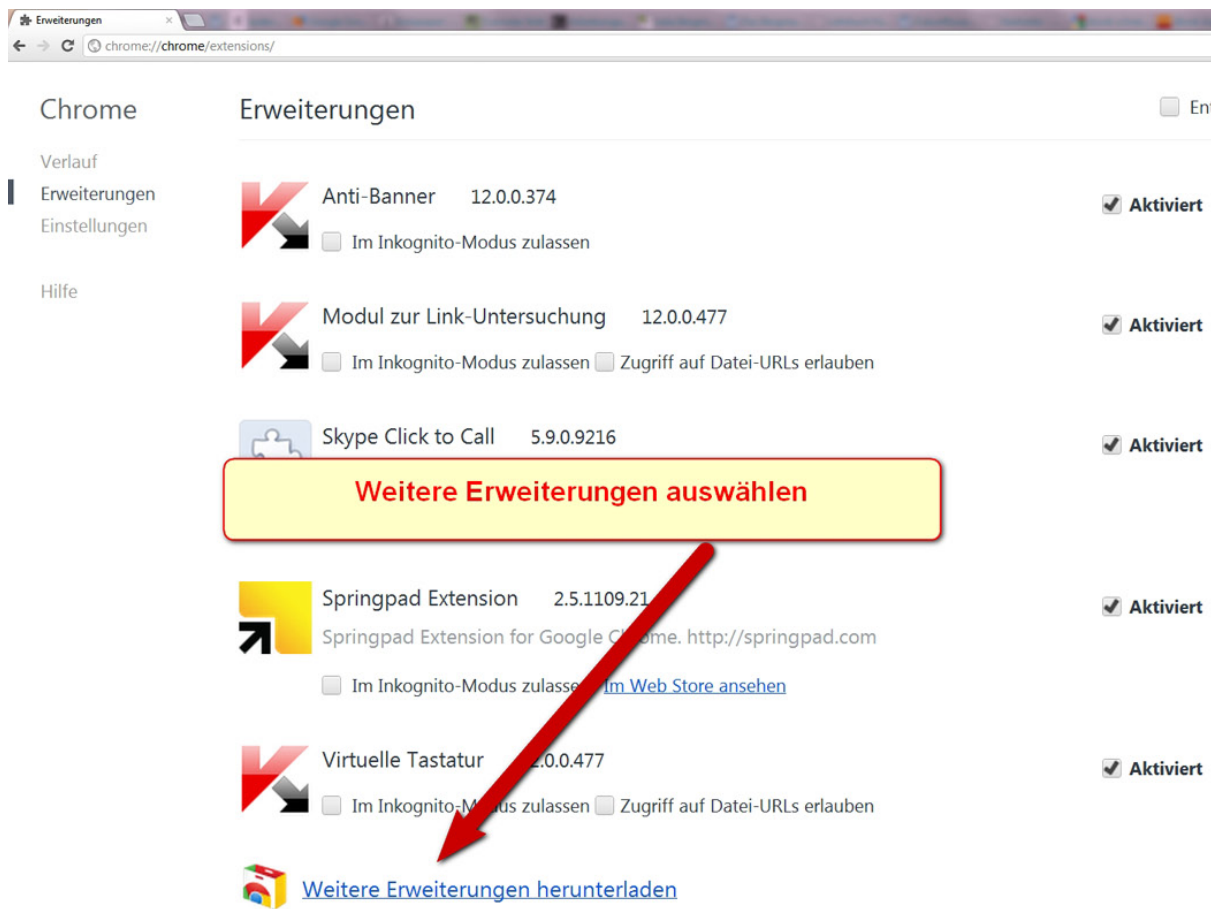


Vorsicht! Beim Chrome gibt es viele Add-ons, die lediglich zu einer bestimmten Webseite weiterleiten. Das ist Etikettenschwindel, denn hier reicht ein Lesezeichen absolut aus.

Empfehlenswerte Chrome-Add-ons

Viele von den oben bei Firefox aufgezählten Add-ons werden auch für den Chrome angeboten.

\section{Musts}

- $\quad$ Adblock Plus - Unterdrückung von Bannerwerbung

- Wikipedia Companion - schnelle Suche in der deutschen oder englischen Wikipedia

- Shareaholic - die aktuelle Seite in verschiedene soziale Dienste einpflegen

- Similar Web - ähnliche Webseiten anzeigen lassen

- Evernote Clearly - Seiten ohne Rauschen lesen

\section{Gute Produktivitätswerkzeuge}

- Delicious oder Diigo - beide sozialen Bookmarkdienste bieten gute Funktionalität

- feedly - RSS-Reader

- TweetDeck oder Silver Bird - Twitter Clients

- Shortcuts for Google - Google-Dienste at your fingertips

- Dilbert Comics - das Lachen über den täglichen Wahnsinn im Büro

- Stealthy - Proxyserver für aktuelle Abfrage wählen

Sie müssen bei den hier vorgestellten Browsern nicht immer mit der Maus arbeiten. Manchmal sind Tastenkombinationen schneller oder auch angenehmer. Wir haben Ihnen hier die „Shortcuts“ aufgelistet, deren Verwendung ein Plus an Effektivität bringt:

Hilfreiche Shortcuts für Internet Explorer, Firefox und Chrome

- $\quad$ F5 - Seite neu laden

- $\quad$ F11 - Umschalten in die/aus der Vollbilddarstellung

- Strg/Ctrl+<auf einen Link klicken> - öffnen der verlinkten Webseite in einem neuen Tab/Registerblatt

- Strg/Ctrl+<+> - größere Darstellung der Webseite/Zoom in

- Strg/Ctrl+<-> - kleinere Darstellung der Webseite/Zoom out

- $\quad$ Strg/Ctrl+Tab - nächsten Tab anzeigen

- $\quad$ Strg/Ctrl+9 - zum letzten Tab wechseln

- $\quad$ Strg/Ctrl+b - Lesezeichen anzeigen

- $\quad$ Strg/Ctrl+e - Suchfeld aktivieren

- $\quad$ Strg/Ctrl+f - Suche in der angezeigten Seite

- $\quad$ Strg/Ctrl+h - Verlauf anzeigen

- Strg/Ctrl+l - markiert den Link der aktuellen Webseite, so dass man inn mit Strg/Ctrl+c gut in die Zwischenablage importieren kann

- $\quad$ Strg/Ctrl+o - öffnet ein Explorer-Fenster, mit dem man eine Datei von der Festplatte auswählen kann, die im Browser geöffnet werden soll

- $\quad$ Strg/Ctrl+p - die Webseite drucken

- $\quad$ Strg/Ctrl+t - neuen Tab öffnen

- $\quad$ Strg/Ctrl+w - aktuellen Tab schliessen
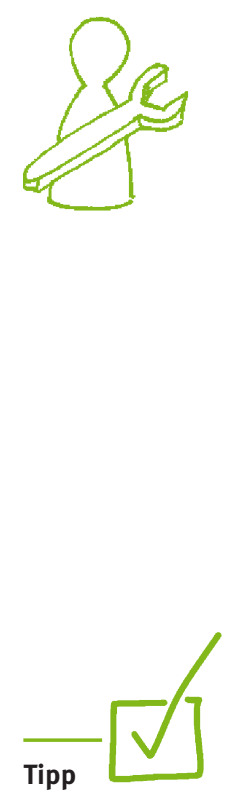

Sie können einzelne Add-ons, die in der Kopfleiste des Browsers angezeigt werden, ganz leicht durch Rechtsklick und das dann erscheinende Aufklappmenü verwalten.

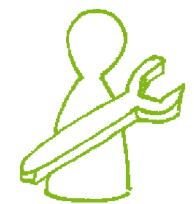

